\title{
La investigación cualitativa como práctica social, histórica y política orientada por principios
}

\author{
I ${ }^{1}$ Fernando Peñaranda, ${ }^{2}$ Miryam Bastidas, \\ ${ }^{3}$ Julio Nicolas Torres, ${ }^{4}$ Gloria Matilde Escobar I
}

Resumen: Este artículo presenta los aprendizajes de una reflexión permanente sobre el método de investigación utilizado en el proyecto "Hacia la construcción de una propuesta educativa para la crianza basada en el diálogo de saberes", realizada en Medellín (Colombia) entre el 2007 y 2009. Inicialmente fue planteado desde una propuesta de Investigación - Acción - Participativa, pero a medida que se fue desarrollando y en respuesta a las demandas del trabajo de campo, a las interacciones con los actores y a los hallazgos, el equipo de investigación necesitó reconstruir y reajustar la propuesta metodológica. Pero más que incluir técnicas de investigación nuevas o modificar las actividades planeadas, se trató de un proceso en el que el método fue ganando nuevos significados. Se fue comprendiendo de una manera más amplia, abierta y flexible en la medida en que se incorporaron aportes de otras perspectivas metodológicas. Sin embargo, siempre hubo una guía que finalmente mantuvo la orientación de la investigación: los principios que se trazaron desde el comienzo y que se concretaron en los productos esperados. Para el equipo de investigación, el método tomó una nueva dimensión desde una perspectiva dialéctica, entendido como un devenir en el marco de una totalidad, donde no puede comprenderse por fuera del objeto de estudio. En este sentido, constituye una práctica social, histórica, cultural y política, planteada desde un marco complejo de negociación, manteniendo una idea de rigor fundada en los principios como una serie de valores que orientan la investigación.

> Palabras clave: Investigación cualitativa, método, rigor, reflexión, principios.

\footnotetext{
1 Universidad de Antioquia, Facultad Nacional de Salud Pública, Colombia. Correo electrónico:

fernandopenaranda@gmail.com

2 Universidad de Antioquia, Facultad de Medicina,

Colombia. Correo electrónico: miryamba@gmail.com

${ }^{3}$ Universidad de Antioquia, Facultad de Medicina, Colombia. Correo electrónico: jnicolastorres@gmail.com

${ }^{4}$ Universidad de Antioquia, Facultad de Odontología Colombia. Correo electrónico gescobarp@gmail.com
}

Recibido: 30/08/2011 Aprobado: 27/12/2011 
Este artículo constituye un esfuerzo por recoger los aprendizajes alcanzados en el marco del proyecto de investigación "Hacia la construcción de una propuesta educativa para la crianza basada en el diálogo de saberes”, realizada en Medellín (Colombia) entre el 2007 y 2009. Nuestro grupo, lleva más de una década investigando y reflexionando sobre el Programa de Crecimiento y Desarrollo en la ciudad de Medellín ${ }^{1}$, está conformado por profesionales del área de la salud interesados en las prácticas de crianza que realizan los adultos con los niños y niñas, además en cómo la educación contribuye a la salud y al bienestar de ambos.

Nuestra experiencia investigativa ha sido desde una perspectiva cualitativa (LINCOLN; GUBA, 2005), fundamentalmente desde la hermenéutica y la etnografía. Siguiendo los cánones convencionales, nos acostumbrados a adoptar un método, y con base en éste plantear un diseño general que orientara el desarrollo de la investigación. Sin embargo, reconocíamos el carácter emergente del método, por lo que los diseños constituían referentes generales sobre los cuales el trabajo de campo se ajustaba a las realidades encontradas. El proceso de análisis se centraba en la codificación y categorización de los datos; ello nos demandaba un gran esfuerzo en tiempo y manejo técnico de los datos, para lo cual nos apoyábamos en programas de software.

Los proyectos nos permitieron acercarnos a los actores del Programa: adultos significativos y miembros de los equipos de salud. Comprendimos su necesidad de compartir inquietudes y angustias propias de su rol, unos en la crianza de los niños y otros como educadores, y decidimos aprovechar esas instancias para reflexionar con ellos en la búsqueda de alternativas de solución a sus problemas particulares. Se hizo evidente lo difícil que resultaba para los miembros de los equipos de salud cambiar sus prácticas pedagógicas, a pesar de contar con los conocimientos y la disposición para hacerlo.

Esta experiencia nos cuestionó sobre nuestro papel como investigadores y el propósito mismo de la investigación. Como académicos, reconocíamos la importancia de la investigación para construir conocimiento útil en el desarrollo de la teoría disciplinar y para la formulación de políticas y programas de salud más pertinentes, pero aquello no era suficiente, queríamos realizar investigaciones que además pudieran apoyar a las personas con las cuales trabajábamos, que les sirvieran en la búsqueda de soluciones a sus problemas particulares.

Fue así como nos embarcamos en una investigación diferente a la que estábamos acostumbrados. Esto constituyó un gran reto, pues se trataba de 
incursionar en un campo inexplorado, y por tanto sujeto a cuestionamientos, incertidumbres y dudas sobre lo que hacíamos y la forma en que lo hacíamos. Fue necesario de-construir muchos de nuestros supuestos sobre el significado de la elección de un método de investigación y su desarrollo metodológico, sobre el análisis de la información, sobre el papel de la reflexión y la praxis. A continuación presentamos nuestras experiencias y el tránsito hacia la transformación de nuestras prácticas y del método mismo.

\section{El proyecto}

Elaboramos un nuevo proyecto para avanzar en la comprensión del Programa de Crecimiento y Desarrollo, esta vez desde un método que ofreciera a sus participantes elementos útiles para la solución de sus problemas particulares y nos permitiera profundizar en las dificultades que entraña el cambio de modelo pedagógico en el sector salud (PEÑARANDA et al., 2006; BASTIDAS et al., 2009). Propusimos una investigación-acción-participativa, con la cual esperábamos generar una propuesta educativa acorde a las necesidades, intereses y características de los actores, basada en el diálogo de saberes.

Esperábamos la participación de los adultos como investigadores de su propia crianza y del proceso educativo, así como algunos mecanismos de apoyo grupal. De otro lado, esta vez no seríamos observadores, tendríamos un doble papel: el de educadores como integrantes del equipo de salud y el de investigadores de nuestra propia práctica educativa; con ello buscábamos avanzar en la comprensión del proceso educativo del programa.

Partimos de un diseño en tres momentos: preparación, desarrollo de la propuesta educativa y sistematización (BOSCO PINTO, 1987). Siguiendo a Reason y Bradbury (2001), establecimos los criterios de calidad y definimos como productos esperados:

- La producción de conocimiento útil para los involucrados

- El logro de nuevas formas de comprender la realidad para todos los actores

- La participación de todos los implicados

- El desarrollo de un proceso emergente y evolutivo, tan importante como los resultados mismos.

Entre febrero y agosto del 2007 se llevó a cabo la preparación interna, centrada en la conceptualización sobre el diálogo de saberes, para apoyar teóricamente la 
construcción y ejecución de la propuesta pedagógica. Revisamos los principales textos de Paulo Freire y de otros educadores populares latinoamericanos. El producto del avance conceptual logrado fue publicado en un artículo sobre la educación para la salud pensada desde el diálogo de saberes (BASTIDAS et al., 2009).

El proyecto fue financiado por el Comité para el Desarrollo de la Investigación de la Universidad de Antioquia y aprobado por el Comité de Ética de Investigación de la institución. Se ajustó a los patrones exigidos por la declaración de Helsinki. No presentó conflicto de intereses. Los adultos significativos participaron voluntariamente y firmaron el consentimiento informado.

El trabajo de campo se llevó cabo en el Programa de Crecimiento y Desarrollo de la institución de salud adscrita a la Universidad de Antioquia, en el que tres de los investigadores participan como profesores de pediatría.Conformamos un grupo de 12 niños y niñas con sus cuidadores o adultos significativos (once madres, ocho padres, cinco abuelas y un abuelo), con ellos realizamos diez sesionesentre agosto de 2007 y octubre de 2008, a partir delprimer mes de vida de los niños y hasta los catorce meses de edad. Unas sesiones se desarrollaron bajo el formato regular del programa, que incluye actividades educativas con los adultos y el monitoreo del crecimiento y desarrollo de los niños y niñas; intercaladas con éstas, realizamos otras en las cuales los adultos significativos reflexionaron con los investigadores sobre la crianza y el proceso educativo vivido.

El análisis y la construcción teórica se realizaron de manera simultánea con la recolección de la información, en dos ámbitos: las sesiones con los adultos significativos, registradas en diarios de campo, y las reuniones del equipo investigador consignadas en actas. De esta manera, el proceso se fundamentó en la praxis como un asunto dialéctico de acción-reflexión, y en la reflexividad como experiencia consciente de los investigadores, mirándonos como sujetos investigadores e involucrados, maestros y educandos, que permitió reorientar permanentemente el desarrollo investigativo (LINCOLN; GUBA, 2005).

\section{La ejecución del proyecto}

Una vez iniciado el trabajo de campo, nos encontramos con la necesidad de modificar los procedimientos de recolección y análisis de la información, para responder a la dinámica de la investigación y del proceso educativo. Había que analizar lo que ocurría en las sesiones con los adultos significativos en relación 
con la crianza y con el proceso educativo, pero también con nuestro rol como educadores, de tal manera que la reflexión y aprendizajes redundaran en cambios en el proceso educativo. La propuesta inicial se quedaba corta para poner en sintonía la investigación con la acción (el proceso educativo).Ello implicaba renunciar a la transcripción, codificación y categorización a la manera tradicional, para orientarnos más hacia el al proceso reflexivo, a la discusión al interior del equipo y a la revisión de la literatura, necesarios para concebir y programar las cambios de nuestra práctica pedagógica.

Esta situación nos generaba inquietud, a la luz de la idea de rigor que habíamos construido en proyectos previos, pero a medida que pasaba el tiempo nos dábamos cuenta de que lo central era el proceso reflexivo(el trabajo en equipo con los hallazgos).Gracias a esta reflexión llegamos al convencimiento de que a pesar de nuestra experiencia investigativa y del trabajo teórico, habíamos comenzado el programa de una manera muy similar a como lo habíamos observado en otros equipos de salud: la educación centrada en transmitir los conocimientos disciplinares, con poca atención a los aportes y conocimientos de los adultos significativos. Ello nos generó frustración, pero reforzó aún más la necesidad de focalizar la atención en la reflexión de nuestra práctica pedagógica (el trabajo teórico nos había mostrado que este era un asunto básico para la transformación de los educadores y de las prácticas educativas).

Regresamos entonces a Freire, ahora con una visión diferente, buscando resolver nuestras incertidumbres sobre como continuar con las actividades educativas, y nos percatamos de un asunto que no habíamos dimensionado antes: su propuesta educativa estaba basada precisamente en un proceso de investigación participativa: "la investigación temática". Desde estos referentes, organizamos nuestra práctica educativa tratando de aplicar su propuesta basada en el análisis de los "temas generadores".

Ala par que pretendíamos darle un nuevo enfoque a nuestra propuesta educativa, nos dimos cuenta de otra situación que cambiaría definitivamente el rumbo de la investigación: no habíamos establecido un ambiente de escucha, con espacios para conocer a los educandos, como debería ser desde una propuesta de diálogo de saberes. Avanzada la investigación, generamos esta instancia, y encontramos que las vidas de estos adultos encerraban dramas, conflictos, expectativas, alegrías y tristezas, en contextos particulares, algo que denominamos "el surgimiento del 
sujeto". Vimos entonces la necesidad de que otros educadores en salud pudieran comprender que la educación a un adulto significativo requiere un acercamiento particular, en tanto no todas las recomendaciones son pertinentes, ni todos los contenidos tienen el mimos significado para todos. Surgió entonces la idea de presentar estas experiencias desde una perspectiva de totalidad, en su valor único, y encontramos en la investigación narrativa una vía para lograrlo. Dado que nuestra experiencia en este tipo de investigación no era muy amplia, debimos hacer un estudio más profundo de este tópico con el apoyo de expertos.

De otro lado, el trabajo teórico desarrollado y su aplicación en nuestra práctica pedagógica empezó a develar nuevos conflictos y problemas: ¿era esta una investigación participativa en la que nosotros teníamos el control sobre el proceso educativo y además los intereses de los adultos significativos eran diferentes a los nuestros? Se hizo evidente que cada uno de los actores, educadores y educandos, tenía su propia agenda e intereses. Sin embargo, la crianza era el punto de encuentro, y aunque nosotros la abordábamos desde una perspectiva teórica y educativa, ellos lo hacían desde sus experiencias y la racionalidad cotidiana para resolver sus problemas.

Al utilizar la propuesta de Freire (1975) para construir los temas generadores, no tuvimos los resultados que esperábamos ante las demandas, necesidades e inquietudes de los adultos significativos y la dinámica del programa, por lo que asumimos una concepción sobre los temas generadores más simple, abierta y flexible. Esta reflexión nos generó otra nueva fase de desconcierto e inquietud en el equipo. ¿Qué tipo de investigación estábamos haciendo finalmente?¿Habíamos perdido el norte? ¿Podríamos presentar ésta como una investigación rigurosa? Con mucha incertidumbre, nos encontrábamos ahora frente a dos problemas simultáneos: no solo teníamos que vérnosla con nuestra práctica como educadores sino también como investigadores (el primero era un asunto que habíamos previsto, pero no así el segundo).

Así que éramos un grupo de educadores que buscaba aprender de nuestra práctica pedagógica, en este orden de ideas, nos encontrábamos en el tipo de investigación que Stenhouse (1979) había descrito como investigación-acción, en la medida que era una investigación articulada con la práctica. Desde este referente teórico pretendíamos construir una práctica pedagógica reflexionada, esto es, crítica, como camino para la transformación de los educadores y 
de su práctica educativa. En este punto surgió la necesidad de profundizar nuestra fundamentación teórica sobre el pensamiento reflexivo más allá de los planteamientos de Freire, para lo cual consultamos investigadores como Hatton y Smith (1995), Dewey (1938) y Schön (1983).

Revisando de nuevo a Stenhouse y su concepción de una investigación articulada con la práctica, llegamos a una nueva forma de comprender nuestra lectura de los teóricos de la sistematización. Al elaborar el diseño habíamos incluido la sistematización como un momento del proyecto, como una instancia para dar cuenta del proceso, que para nosotros era un asunto central. Nos pareció en ese entonces un tanto forzada la idea de concebir la sistematización como un método de investigación, tal como lo plantean algunos académicos latinoamericanos, entre ellos Marco Raúl Mejía (2007). Pero ahora que estábamos embarcados en estas dificultades y que la práctica investigativa se nos convertía en algo complejo, reconocimos que también podíamos concebir esta investigación desde la sistematización como una vertiente investigativa.

En este punto, a pesar de que el significado del método había sufrido transformaciones continuadas la idea que teníamos de una investigación al servicio de los actores-que ahora incluía también nuestra práctica investigativa- y de la relación entre la investigación y la acción para transformar la realidad y los sujetos inmersos en ella, se mantenía vigente.

\section{Discusión}

El procedimiento que seguimos para llevar a cabo la investigación, fue el resultado de una serie de ajustes continuados, en respuesta a las demandas procedentes de los propósitos trazados, del proceso de educación/investigación que llevábamos a cabo, y de la reflexión teórica permanente sobre nuestra práctica investigativa (método) y educativa (objeto), realizada de manera completamente articulada e interdependiente.

Pero más allá de cambiar los procedimientos (que poco cambiaron a lo largo del proyecto), lo que realmente se fue transformando de manera paulatina, fue nuestra comprensión de lo que estábamos haciendo y como lo estábamos haciendo, lo cual nos remitía a los propósitos de la sistematización (JARA, 2010; MEJÍA, 2007).

En este punto se hace necesario para nosotros responder las preguntas que nos hicimos con respecto al método, su rigor, y en general sobre los supuestos 
y criterios que sustentan la calidad de nuestra investigación. Entonces, ¿qué método finalmente seguimos? ¿Qué concepción de método subyace a nuestros procedimientos investigativos? ¿Qué rigor tiene esta investigación?

Para dar respuesta a estas inquietudes, es necesario analizar en primera instancia la concepción hegemónica sobre el método en la investigación proveniente de Descartes, cuya influencia sigue vigente en el campo científico: "[...] entiendo por método reglas ciertas y fáciles, mediante las cuales el que las observe exactamente no tomará nunca nada falso por verdadero, y, [...] llegará al conocimiento verdadero" (DESCARTES, 1989, p. 79).

Esta idea de método ha sido cuestionada desde varias orillas epistemológicas. Adorno (2006) ha utilizado la expresión marxista de "fetiche" para referirse a dicha concepción de método como la "cosificación" de una práctica social que pretende ocultar sus determinaciones históricas, políticas y sociales. Afirma este autor que así entendido, el método se le impone al objeto, un absurdo en tanto la primacía la debe tener el objeto, y puede suceder entonces que el método se convierta en camisa de fuerza para encontrar o resolver lo verdaderamente pertinente y necesario. No entendemos la idea de primacía del objeto como en algunas corrientes de investigación positivista - en especial la epidemiología clásica - para las cuales las características del objeto determinan el tipo de método a utilizar, sino desde perspectivas como la de Janisek (1994), quien se opone a la "metodolatría" porque el énfasis dado al método y a la trinidad compuesta por validez, confiabilidad y generalización, se puede convertir en un obstáculo para la comprensión de la experiencia de los actores, e invita a centrarse en lo sustancial: los hallazgos.

Gadamer (2006) también encuentra problemática la idea cartesiana que asocia método a verdad; a lo sumo, esta podría equipararse con certeza. Esta visión dejaría por fuera aquello que no podemos determinar con certeza, pero que aún así es verdad, en la medida que nuestras posibilidades de encontrar la verdad son finitas. Más aún, Gadamer expresa desconfianza hacia los trabajos que - respondiendo a una moda- presentan con gran detalle el método, porque más bien dan la idea de imitar y no de estar en la búsqueda de algo nuevo: "[...] los resultados más importantes y fecundos alcanzados en las ciencias del espíritu quedan muy al margen del ideal de verificabilidad" (GADAMER, 2006, p. 57). En esto concuerda Adorno (2006) al afirmar que la verdad como certeza se puede convertir en talanquera: 
[...] creo que esta sobrestima del método en sí, es decir, de la confiabilidad del mé-

todo, separada del interés por el tema específico [...] es el resultado del temor a la inseguridad y que con tal de poseer algo seguro en la mano, olvidan la relevancia, el contenido, la sustancia de aquello a lo cual lo seguro se refiere [...] En oposición a este ideal [la seguridad], me atrevería a conjeturar que el conocimiento solamente es productivo cuando va más allá del juicio puramente analítico [...] no existe ninguna verdad [...] que no esté expuesta al riesgo de ser falsa. (ADORNO, 2006, p. 104-105).

La idea de la primacía del objeto (el tema), se relaciona con los planteamientos de Kincheloe y Mclaren (2005) cuando hablan sobre el método como un proceso de negociación que para nuestro caso fue un proceso de negociación entre los aspectos teóricos, prácticos y culturales. Estos autores, recogiendo la idea de Denzin y Lincoln (2005) sobre el investigador como "bricoleur", avanzan sobre una conceptualización de método como un procedimiento emergente en la medida en que se va construyendo durante el desarrollo de la investigación; para ello el investigador debe tomar de diferentes técnicas investigativas, en respuesta a las necesidades que la investigación demanda. Constituye

[...] un proceso cognitivo de alto nivel que implica construcción y reconstrucción, diagnostico, negociación y reajuste [... por lo cual el método se entiende como] una tecnología de justificación, esto es, una forma de defender lo que afirmamos que hacemos y el proceso por el que lo sabemos. (KINCHELOE; MACLAREN, 2005, p. 317-318).

Por su parte, Feyerabend (1975) plantea una perspectiva de ciencia que reconoce que las teorías producen el dato, y entiende el método -la acción investigativafundamentalmente como una actividad creativa con una gran dosis de intuición. En este sentido, la realidad solo puede comprenderse desde múltiples perspectivas (teorías) y "la elección entre teorías que son suficientemente generales para proporcionar una concepción del mundo comprehensiva y entre las que no hay conexión empírica puede llegar a ser una cuestión de gustos" (FEYERABEND, 1975, p. 134). Más aún, Feyerabend propone liberarnos de la idea de ciencia como resultado de reglas fijas, "recordando que la separación existente entre las ciencias y las artes es artificial, que es el efecto lateral de una idea de profesionalismo que deberíamos eliminar" (FEYERABEND, 1975, p. 136). Bien nos dice este físico epistemólogo que la ciencia no se puede desligar de sus asuntos afectivos y estéticos, sin los cuales no sería imaginable cualquier producción humana. Así que la investigación no es un asunto neutral, pues está orientada desde nuestros "prejuicios" (GADAMER, 2007a), que incluyen posiciones teóricas, políticas y culturales, de raza, sexo e incluso edad (DENZIN; LINCOLN, 2005). 
Una pregunta que habría que hacerse en este momento sería si la idea de investigación y de método es una producción de la modernidad. La respuesta requiere recoger la preocupación que se encuentra ya desde Platón por desligar la opinión (doxa) del conocimiento científico (episteme). Aristóteles (CORTINA, 1998) propone un método centrado en el diálogo y la reflexión, método que recogió de Platón en sus diálogos y que no hace referencia a una serie de pasos o de procedimientos, y más bien se entiende como una actitud crítica, en un ambiente donde se debaten las ideas contrarias o se busca la coherencia de los argumentos en el marco de una discusión racional (GADAMER, 2007b). Esta búsqueda de coherencia y de sustento racional cubre no solo el discurso de los otros sino el propio, y así Platón en el Parménides pone a prueba sus propias tesis en el marco de un proceso reflexivo y de autocrítica.

Una idea de investigación similar podemos encontrar en Dewey (1938), quien plantea el pensamiento reflexivo como un pensamiento investigativo, que surge cuando necesitamos resolver problemas de la práctica. Tanto Freire (1975) como Stenhouse (1979), recogen la reflexión como la base para el aprendizaje que logra el educador/investigador en su práctica pedagógica.

Pero la crítica que se hace a la idea hegemónica de método, requiere un elemento adicional que aporta Bourdieu (FERNÁNDEZ, 2004). Para Bourdieu, la ciencia es un escenario jerarquizado en el cual existen intereses diferentes, en la medida en que se han configurado comunidades académicas diversas y en ocasiones contrapuestas, cuyas relaciones de poder tienen consecuencias sobre las propias comunidades y sobre el accionar de los investigadores. En este escenario, la concepción de método y su relación con el establecimiento de criterios sobre la calidad de las investigaciones se debate en un ámbito de intereses y visiones divergentes, donde el poder juega un papel importante para establecer la legitimidad en cuanto a estos asuntos (DENZIN; GIARDINA, 2008).

Con las referencias teóricas anteriormente presentadas y con un proceso adicional de reflexión, comprendemos ahora el método más allá de una serie de procedimientos, inclusive más allá de una forma de pensar o de ver desde una perspectiva teórica como lo entienden Geertz (1993) y Wolcott (1999). Serían entonces tres los puntos centrales dentro de la concepción de método que ahora sostenemos: el primero, es comprender el método fundamentalmente como una actitud, como una posición ante el conocimiento basada en la crítica, la cual 
a su vez se sustenta en el proceso de reflexión. La crítica implica también la autocrítica, necesaria para superar los dogmatismos y estar abiertos al cambio.

El segundo punto consiste en reconocer el método como una práctica social, con sus determinaciones histórico-sociales, culturales y políticas. Entendemos entonces el método en el marco de una praxis - práctica reflexionada a la luz de la teoría (FREIRE, 1975), que implica un proceso de negociación de las demandas de orden interno y externo. Las internas hacen referencia a unos ideales de investigación (valores) y a unos propósitos que siguen esos valores, en relación con supuestos de orden teórico, epistemológico, político y personal-afectivo de los investigadores, esto es, con los principios que rigen la investigación. Las demandas de orden externo provienen del contexto sociocultural y político en el cual se lleva a cabo la investigación, de los resultados emergentes, de la interacción con los actores, de la teoría que aporta nuevas visiones, y de todas las demandas institucionales que configuran el campo de la ciencia.

De todas maneras, la negociación estaría guiada por los principios como conjunto de valores que sustentan la investigación, y para el caso del presente proyecto de investigación al cual hacemos referencia, los principios que recogimos de Reason y Bradbury (2001) y que materializamos en los productos esperados, se mantuvieron siempre vigentes, como guías de orientación de la acción, la reflexión y la teorización, a pesar de que el significado del método fue modificándose a través del proceso.

El método como práctica social requiere entenderse como un proceso en construcción en una relación dialéctica con el objeto: método y objeto constituyen los polos dialécticos necesarios para la identidad de cada uno (KOHAN, 2003; DE LA GARZA, 1986). Por lo tanto, los significados que el método y sus componentes adquieren, son un producto de su devenir, dentro de una totalidad que implica su relación dialéctica con el objeto y demás determinaciones sociohistóricas y culturales. En este sentido, es pertinente el proceso de reconstrucción que la sistematización provee para comprender el método dentro de una totalidad.

El tercer punto hace referencia al carácter creativo del proceso investigativo, que implica imaginación, pero que también, como lo plantea Feyerabend (1975), tiene que ver con los gustos, con los sentimientos y con una mirada estética. De esta manera, no entendemos el rigor en el marco de una serie de pasos y procedimientos, que más bien pueden convertirse en estorbo para la investigación. 
1202 El rigor relacionado con principios, como una serie de valores que orientan la investigación, desde posiciones epistemológicas y políticas determinadas, tendría que ver con la capacidad del investigador para hacer explícitos sus supuestos epistemológicos, teóricos y políticos en el marco de un proceso de reflexión y autocrítica que evidencie su transformación para comprender su praxis investigativa. Así, el rigor hace referencia a la capacidad del investigador para dar cuenta del proceso vivido en la investigación, para mostrar el método como devenir en el marco de sus determinaciones como totalidad concreta. Pero también remite a la capacidad para evidenciar su actuación conforme a los principios asumidos y a las comprensiones logradas.

\section{Conclusiones}

La experiencia investigativa que presentamos nos llevó a comprender la investigación más allá de la concepción hegemónica de método. Desde una perspectiva dialéctica, el método como devenir en el marco de una totalidad no pude comprenderse por fuera del objeto de estudio. Entendimos entonces el método como una práctica social, histórica, cultural y política, planteada desde un marco complejo de negociación teórica-epistemológica, política y cultural. Negociación orientada por una posición crítica y reflexiva del investigador, que responde a sus valores en el orden teórico, epistemológico, político, cultural, afectivo y estético, por lo cual hemos denominado a la nuestra, una investigación "por principios".

Orientar la investigación desde una perspectiva en el orden de los principios, requiere a su vez que el investigador fortalezca su autonomía investigativa, su capacidad reflexiva y su autoconciencia, fundamentales para construir propuestas investigativas creativas, pertinentes y comprometidas con la justicia social y con la solución de los problemas de los actores vinculados a la investigación. Esto hace referencia a la capacidad de tomar decisiones que conllevan riesgos, dentro de un campo de investigación surcado por jerarquías que ejercen poder para establecer los criterios sobre la legitimidad de lo que cuenta como método.

Consideramos que esta idea de método permite trascender la "metodolatría" (JANISEK, 1994) para concentrarnos en los hallazgos fundamentales, de manera que finalmente el método se encuentre al servicio de la investigación y no al contrario, y que la práctica investigativa sirva para actualizar, expresar y cristalizar 
los deseos y las emociones de investigadores comprometidos en promover valores como la democracia, la justicia social y la solidaridad.

\section{Referencias}

ADORNO, T. Introducción a la Sociología. Barcelona: Gedisa, 2006.

BASTIDAS, M.; PÉREZ, N.; TORRES, N. et al. La comprensión de los significados que del programa de crecimiento y desarrollo tienen sus actores: un paso hacia su cualificación. Revista Ciencia y Salud Colectiva, Rio de Janeiro, v. 14, n. 5, p. 1919-1928, 2009.

BOSCO PINTO, J. La investigación-acción. Manizales: Agencia Canadiense para el Desarrollo Internacional, 1987.

CORTINA, A.; MARTíNEZ, E. Ética. Madrid: Ediciones Akal, 1998.

DE LA GARZA, E. Hacia una metodología de la reconstrucción. Fundamentos, crítica y alternativas a la metodología y técnicas de investigación social. México: UNAM, 1998.

DENZIN, N., GIARDINA, M. Introduction: The elephant in the living room, or advancing the conversation about politics of evidence. In: DENZIN, N. y GIARDINA, M (Orgs.). Qualitative inquiry and the politics of evidence. Walnut Creek: Left Coast Press, 2008. p. 9-51. DENZIN, N.; LINCOLN, Y. Preface. In: DENZIN, N.; LINCOLN, Y. (Orgs.). The Sage handbook of qualitative research, ( $3^{a}$ ed.). Thousand Oaks: Sage Publications, 2005. p. IX-XIX.

DESCARTES. Reglas para la dirección del espiritu. Madrid: Alianza, 1989.

DEWEY, J. Logic. The theory of inquiry. New York: Henry Holtand Company, 1938.

FERNÁNDEZ, J. M. Interdisciplinariedad en ciencias sociales: perspectivas abiertas por la obra de Pierre Bourdieu. Cuadernos de Trabajo Social, Madrid, v. 17, p. 169-193, 2004.

FEYERABEND, P. Contra el método. Barcelona: Ariel, 1975.

FREIRE P. Pedagogía del oprimido.México: Siglo XXI editores, 1975.

GADAMER, H. G. Verdad y método I. Salamanca: Sígueme, 2007(a).

GADAMER, H. G. La idea de lógica de Hegel. En: La dialéctica de Hegel. 7 ed. Madrid: Ediciones Cátedra, 2007(b). p. 75-108.

GADAMER, H. G. Verdad y método II. Salamanca: Sígueme, 2006.

GEERTZ, C. Descripción densa: hacia una teoría interpretativa de la cultura. En: BOHANNAN, P. y GLAZER. M. (Orgs.). Antropologia: Lecturas. 2 ed. Madrid: McGrawHill, 1993. p. 547-568.

HATTON, N.; SMITH, D. Reflection in teacher education: towards definition and implementation. Teaching and Teacher Education, v. 11, n. 1, p. 33-49, 1995.

JANISEK, V. The dance of qualitative research design: Metaphor, methodolatry, and meaning. In: Denzin, N.; Lincoln, Y. (Orgs.). Handbook of qualitative research. London: Sage, 1994. p. 209-219. 
JARA, O. El desafío politico de aprender de nuestras prácticas. Disponible en: <http://www. cepalforja.org/sistem/documentos/aprenderdepracticas.pdf>. Acesso em: 10 dic 2010.

KINCHELOE, J.; MCLAREN, P. Rethinking critical theory and qualitative research. In: DENZIN, N.; LINCOLN, Y. (Orgs.). The Sage handbook of qualitative research, (3a ed.). Thousand Oaks: Sage, 2005. p. 303-342

KOHAN, N. Marx en su (tercer) mundo. 2 ed. La Habana: Centro de Investigación y Desarrollo de la Cultura Cubana Juan Marinello, 2003.

LINCOLN, Y.; GUBA, E. Paradigmatic controversies, contradictions and emerging confluences. In: DENZIN, N.; LINCOLN, Y. (Orgs.). The Sage handbook of qualitative research. 3 ed. Thousand Oaks: Sage, 2005. p. 191-215.

MEJÍA, M. R. La sistematización como proceso investigativo. O la búsqueda de la episteme de las prácticas. Revista Internacional Magisterio, Bogotá, v. 33, p. 1-17, 2007.

PEÑARANDA, F.; BASTIDAS, M.; ESCOBAR, G. et al. Análisis integral de las prácticas pedagógicas de un programa educativo en Colombia. Salud Pública de México, Morelos, v. 48, n. 3, 229-235, 2006.

REASON, P.; BRADBURY, H. Introduction: Inquiry and participation in search of a world worthy of human aspiration. In: Reason, P. y Bradbury, H. (Orgs.). Handbook of action research. London: Sage, 2001. p. 1-14.

SCHÖN, D. The reflective practitioner. How professionals think in action. New York: Basic Books, 1983.

STENHOUSE, L. Research as a Basis for Reaching. London: Heineman Educational Books, 1985. WOLCOTT, H. Ethnography: A way of seeing. Walnut Creek: Alta Mira Press, 1999.

\section{Nota}

${ }^{1}$ a) Evaluación de la implementación del Programa de Crecimiento y Desarrollo en el escenario de la ley 100 (1999-2000); b) comprendiendo el proceso educativo en el Programa de Crecimiento y Desarrollo (2000-2002); c) hacia la comprensión de los efectos e impacto de la educación en el Programa de Crecimiento y Desarrollo en cuatro instituciones prestadoras de servicios de salud en Medellín (2002-2005); d) hacia la comprensión de la construcción del significado de crianza en el Programa de Crecimiento y Desarrollo (2002-2006). 


\section{Qualitative research as a social, historical and political practice guided by principles}

This paper presents the learning products of a permanent reflective process about the research method used in the project "Towards a construction of an educational proposal of child rearing founded in a dialog of knowledge", conducted in Medellín (Colombia) from 2007 to 2009. At the beginning it was defined a Participatory Action Research method, but as the project was developed and responding to field demands, interactions between actors and to findings, the research team was forced to adjust the methodological approach. This change went beyond including new research techniques or modifying activities. It was a process in which the method gained new meaning. We started to understand it in a more open and flexible way, as we went on taking contributions from different methodological perspectives. But there was a guide that we kept as a reference for adjusting the method: principles settled at the beginning of the project, which were written as expected outcomes. From a dialectic perspective, the research method was understood as "becoming" in a context of totality, where it cannot be understood outside the study object. In this way, method is conceived as a social, historical and political practice, which demands a complex negotiation process, under an idea of rigor, founded in principles as a set of values that guide research.

> Key words: qualitative research, method, rigor, reflection, principles. 